\title{
Heterogeneous Intramolecular Electric Field as a Descriptor of Diels-Alder Reactivity
}

\author{
Matthew R. Hennefarth ${ }^{\dagger}$ and Anastassia N. Alexandrova ${ }^{*,+, \dagger}$ \\ $\dagger$ Department of Chemistry and Biochemistry, University of California, Los Angeles, 607 \\ Charles E. Young Drive East, Los Angeles, CA 90095-1569, USA \\ $\ddagger$ California NanoSystems Institute, University of California, Los Angeles, 570 Westwood \\ Plaza, Los Angeles, California 90095-1569, USA \\ E-mail: *ana@chem.ucla.edu \\ Phone: +1 310 825-3769
}

\begin{abstract}
External electric fields have proven to be an effective tool in catalysis, on par with pressure and temperature, affecting the thermodynamics and kinetics of a reaction. However, fields in molecules are complicated heterogeneous vector objects, and there is no universal recipe for grasping the exact features of these fields that implicate reactivity. Herein, we demonstrate that topological features of the heterogeneous electric field within the reactant state, as well as of the quantum mechanical electron density a scalar reporter on the field experienced by the system - can be identified as rigorous descriptors of the reactivity to follow. We scrutinize specifically the Diels-Alder reaction. Its 3-D nature and the lack of a singular directionality of charge movement upon barrier crossing makes the effect of the electric field not obvious. We show that the electric field topology around the dienophile double bond, and the associated changes in the topology of the electron density in this bond are predictors of the reaction barrier. They are also the metrics by which to rationalize and predict how the external
\end{abstract}


field would inhibit or enhance the reaction. The findings pave the way toward designing external fields for catalysis, as well as reading the reactivity without an explicit mechanistic interrogation, for a variety of reactions.

\section{Introduction}

External electric fields, $\mathbf{E}_{\text {ext }}(\mathbf{r})$, have been shown to play a powerful role in redox chemistry, ${ }^{1}$ enzyme catalysis, ${ }^{2,3}$ heterogeneous catalysis, ${ }^{4-6}$ and zeolite catalysis. ${ }^{7}$ In general, electric fields should be able to influence the kinetics of any reaction that includes reagent polarization during transition state (TS) crossing by stabilizing or destabilizing charged-separated resonance contributors. ${ }^{8}$ External electric fields break the canonical idea that a catalyst has to be a reaction-specific chemical entity, e.g. a molecule or surface. It is even hypothesized by Warshel that enzymes operate primarily under electrostatic preorganization: the idea that the protein scaffold that does not directly participate in the reaction imparts an optimally oriented electric field by which to increase the enzyme's efficiency. ${ }^{2,9,10}$ This effect has been extensively studied experimentally and theoretically in the Ketosteroid Isomerase protein. ${ }^{3,11-21}$ Computational studies on external electric field catalysis have also shown that external fields can dictate stereoselectivity of reactions. ${ }^{22-24}$ Further, within the cytochrome P450 enzyme, an oriented electric field can switch the enzyme from catalyzing a C-H hydroxylation to a $\mathrm{C}=\mathrm{C}$ epoxidation. ${ }^{1}$ That is, by just switching the direction of the field along the $\mathrm{Fe}=\mathrm{O}$ bond, the reaction flips from hydroxylation to epoxidation. External electric fields have also been predicted to drive polar molecules to aggregate through enhanced H-bonding and $\pi$-stacking. ${ }^{25}$ Understanding the role that electric fields can play in catalysis and learning how to control them is of great interest.

Many useful reactions involve rearrangements in three dimensions (3-D), with electrons relocating in multiple areas (aka arrow-pushing), and thus without an obvious singular direction along which to apply an electric field for catalysis. The Diels-Alder reaction is one of them. It is a powerful organic reaction that, in a single concerted step, forms two carbon- 
carbon single bonds with a total of four stereocenters. Despite the complexity, computational studies indeed showed that external fields can control its barrier, stereospecificity, and endo/exo selectivity. ${ }^{8,22,24,26,27}$ In particular, Shaik has shown that $\Delta E^{\ddagger}$ of the Diels-Alder reaction can be affected by a rather strong electric field $(\sim 50 \mathrm{MV} / \mathrm{cm})$ along the direction of bonds formation (z-direction in Scheme 1), via modulating the degree of charge transfer at the TS. ${ }^{24}$ Note that, in these studies, the starting orientations of the reactants was fixed. In reality, a large external field causes the pre-existing and induced dipoles in the system to align with the field. Returning to the reactive configuration, then, comes with an entropic penalty, and decoupling this part of $\Delta S^{\ddagger}$ from the pure effect of the field on $\Delta G^{\ddagger}$ and the reaction rate becomes non-trivial. The problem was circumvented elegantly by Coote et al., ${ }^{28}$ who attached the reactants to a surface and a STM tip and applied an oriented external electric field across the Diels-Alder reaction. A five-fold increase in the reaction rate was measured. ${ }^{28}$ Outside of such an involved setup, fields that can be realistically applied to a reaction without causing reagent reorientation need to be milder. For example, an enzyme scaffold would exert a field on order of $\sim 10 \mathrm{MV} / \mathrm{cm}$, which is at least an order of magnitude smaller than the field produced by the active site itself. ${ }^{12,29,30}$

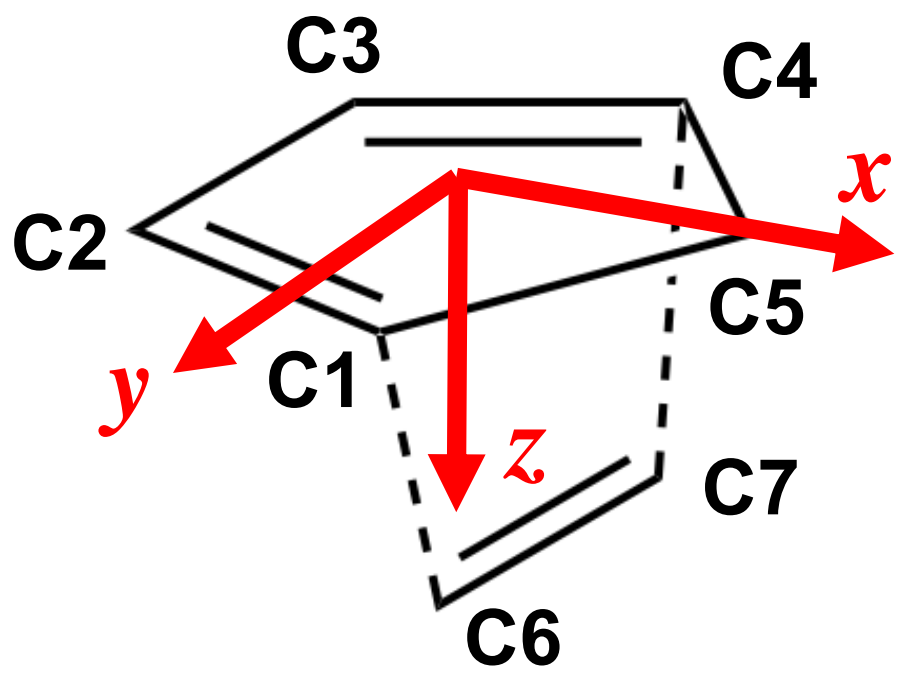

Scheme 1: Orientation, as considered in this study, ( $x, y$, and $z$ direction) within the Diels-Alder reaction between cyclopentadiene and ethylene. 
Note that a field, $\mathbf{E}(\mathbf{r})$, experienced by atoms in a molecular system is a complicated heterogeneous vector object. Electrons (or electron density, $\rho(\mathbf{r})$ ) and nuclei in the system themselves contribute to the majority of this field, $\mathbf{E}_{\text {int }}(\mathbf{r})$, which in turn affects $\rho(\mathbf{r})$ and nuclear coordinates. Of course, $\mathbf{E}_{\text {int }}(\mathbf{r})$ is also a part of the quantum mechanical wave function. The external field, $\mathbf{E}_{\text {ext }}(\mathbf{r})$, which is applied to a system acts as a perturbation. The overall portrait of the intramolecular field is therefore by far more intricate than a uniform vector, and this is likely important for reactions where more than one local dipole is created and more than one bond is shifted. Majority of interesting organic reactions belong to this class. Generally, because the field is largely a result of the system geometry, plus $\mathbf{E}_{\text {ext }}(\mathbf{r})$, its signature can be found in $\rho(\mathbf{r})$, and should be reflected in reactivity. This can be shown most conveniently when nuclear coordinates are restrained or minimally affected by the field, for example in enzymes. ${ }^{12,29}$

In this work we offer a way to detect, analyze, and compare the full heterogeneous intramolecular vector fields, $\mathbf{E}(\mathbf{r})$, (with or without $\mathbf{E}_{\text {ext }}$ ) for the Diels-Alder reaction free of restraints. Three variations of the Diels-Alder reaction are considered: cyclopentadiene with ethylene, 1-methoxycyclopentadiene with cyanoethylene, and cyclopentadiene with maleic anhydride (Scheme 2); which sample a range of polarities. We show that, while the intramolecular field is complicated, features in it can be identified such that they can serve as descriptors of reactivity, even for the apolar version of the reaction (Scheme 2a). We derive field-based descriptors, which allow predicting the reaction barrier, $\Delta G^{\ddagger}$, without an actual calculation of the TS. This result testifies that the intramolecular field is a fundamental metric of reactivity, and brings a great convenience to computation of reaction mechanisms, let alone providing a rigorous descriptor set for PCA or machine learning. In addition, because $\mathbf{E}(\mathbf{r})$ and $\rho(\mathbf{r})$ are interdependent, features in $\rho(\mathbf{r})$, in geometric correspondence with the important features of $\mathbf{E}(\mathbf{r})$, are also reactivity predictors. 
a)

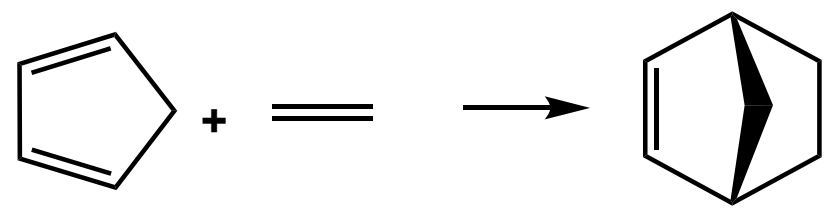

b)<smiles>C=CC#N</smiles><smiles>CO[C@]12C=C[C@@H](C[C@H]1C)[C@H](C#N)C2</smiles>

c)

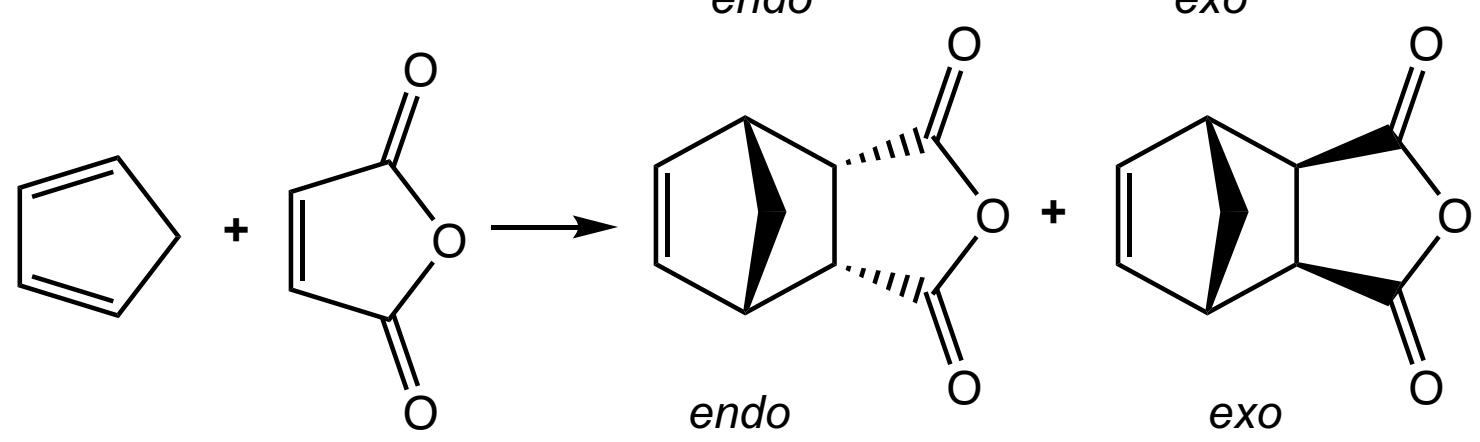

Scheme 2: The three Diels-Alder reactions considered in this study: (a) non-polar reaction of cyclopentadiene and ethylene; (b) polar reaction of 1-methoxycyclopentadiene and cyanoethylene; and (c) moderately polar reaction of cyclopentadiene and maleic anhydride polar. 


\section{Computational Methods}

All calculations were performed in TURBOMOLE 6.6. ${ }^{31-41}$ Geometries were optimized with the B3LYP functional ${ }^{42-45}$ and the def2-SVP basis set. ${ }^{46}$ Note that B3LYP has been shown to perform well for Diels-Alder reactions; however, it has also been shown to overestimate barriers for polar cycloadditions. ${ }^{47}$ Numerical frequency calculations were performed at the same level of theory to confirm the presence of either 0 imaginary frequencies (reactant/product state) or 1 imaginary frequency (TS). Final electronic energies were evaluated with a larger triple- $\zeta$ with polarization (def2-TZVP) basis set. ${ }^{48}$ We used the harmonic approximation from the frequency calculations to calculate the free energy of the system. Implicit solvation was modeled using the conductor-like screening model (COSMO) ${ }^{49}$ with the dielectric constant set to 80 to mimic that of a polar solvent such as water. The external electric field, $\mathbf{E}_{\text {ext }}$, applied to the Diels-Alder reaction varied in strengths from -0.002 to +0.002 a.u. ( 1 a.u. is $51.4 \mathrm{~V} / \AA$ ). All the results discussed are for the field applied in the $z$-direction (Scheme 1), as it has the strongest effect on the reaction from the findings by Shaik. ${ }^{24}$ Our maximum field applied is roughly $\pm 10 \mathrm{MV} / \mathrm{cm}$, again, inspired by enzymes. ${ }^{12,29,30}$

Topological analysis of $\mathbf{E}(\mathbf{r})$ (by $\mathbf{E}(\mathbf{r})$ we mean either $\mathbf{E}_{\text {int }}(\mathbf{r})$ in the absence of $\mathbf{E}_{\text {ext }}$, or $\mathbf{E}_{\text {int }}(\mathbf{r})+\mathbf{E}_{\text {ext }}$ when $\mathbf{E}_{\text {ext }}$ is present) was performed similarly to our previous study, ${ }^{29}$ using the global distribution of stream lines first proposed by Dinh and $\mathrm{Xu}$ in the field of fluid mechanics. ${ }^{50}$ In brief, we apply a grid to discretize the electric vector field in the molecular regions of interest $\left(2 \times 2 \times 4 \mathrm{Bohr}^{3}\right.$ in volume), with $\mathbf{E}(\mathbf{r})$ calculated from the wavefunction in TURBOMOLE using Equation 1.

$$
\mathbf{E}(\mathbf{r})=-\int d \tau^{\prime} \frac{\rho\left(\mathbf{r}^{\prime}\right)\left(\mathbf{r}-\mathbf{r}^{\prime}\right)}{\left|\mathbf{r}-\mathbf{r}^{\prime}\right|^{3}}+\sum_{i} \frac{Z_{i}\left(\mathbf{r}-\mathbf{R}_{i}\right)}{\left|\mathbf{r}-\mathbf{R}_{i}\right|^{3}}+\mathbf{E}_{e x t}
$$

We then randomly sample two points that lie on the same streamline, $\alpha$, and compute the 
mean curvature, $\kappa$ (Equation 2), and Euclidean distance between these two points.

$$
\kappa=\frac{\left|\alpha^{\prime} \times \alpha^{\prime \prime}\right|}{\left|\alpha^{\prime}\right|^{3}}=\frac{\left|\mathbf{E}(\mathbf{r}) \times \mathbf{E}^{\prime}(\mathbf{r})\right|}{|\mathbf{E}(\mathbf{r})|^{3}}
$$

For each region, we sample 100,000 points, and construct 2D histograms of mean curvature versus Euclidean distance. We then use the $\chi^{2}$ metric to compute the distance between each pair of histograms. The $\chi^{2}$ distance between two, $N$-bin, normalized histograms $f$ and $g$ is given by Equation 3.

$$
\chi^{2}: D(f, g)=\frac{1}{2} \sum_{i=1}^{N} \frac{(f[i]-g[i])^{2}}{f[i]+g[i]}
$$

The range of the $\chi^{2}$ metric is $[0,1]$ with values close to 0 representing topologically similar vector fields, and values close to 1 representing topologically distinct vector fields.

The Quantum Theory of Atoms in Molecules (QTAIM) analysis of the electron density was performed using the AIMALL software. ${ }^{51}$ QTAIM is a mathematically rigorous formalism that partitions $\rho(\mathbf{r})$ into atomic basins that are defined by zero-flux surfaces, i.e. surfaces such that the normal vector at any point on the surface, $\mathbf{n}(\mathbf{r})$, satisfies Equation $4 .{ }^{52-55}$

$$
\nabla \rho(\mathbf{r}) \cdot \mathbf{n}(\mathbf{r})=0
$$

There are 4 types of critical points of $\rho(\mathbf{r})\left(\mathbf{r}_{c}\right.$ such that $\left.\nabla \rho\left(\mathbf{r}_{c}\right)=0\right)$ that are dependent on the curvature of $\rho\left(\mathbf{r}_{c}\right)$ : nuclear critical points, bond critical points (BCP), ring critical points (RCP), and cage critical points (SI Figure S4). BCP are saddle points on a ridge in $\rho(\mathbf{r})$ that is minimized along the bond direction, but maximized in all other directions. RCP are saddle points within a ring of nuclear critical points such that $\rho(\mathbf{r})$ is minimized in 2 spatial directions, but maximized in one (direction orthogonal to the ring plane). At each found BCP and RCP, we compute $\rho(\mathbf{r}), \nabla^{2} \rho(\mathbf{r})$, the electrostatic potential $(V(\mathbf{r}))$, and the Lagrangian electronic kinetic energy density $(\mathcal{G}(\mathbf{r})) .{ }^{53}$ In addition, at each BCP, we compute the delocalization index between the two atoms in the bond $(\delta(A, B))$, which measures the 
number of delocalized electrons in that bond. ${ }^{56}$

\section{Results and Discussion}

First, the reaction profiles without $\mathbf{E}_{\text {ext }}$ were computed (Figure 1). For the reaction between non-polar cyclopentadiene and ethylene, the calculated barrier is $26.8 \mathrm{kcal} / \mathrm{mol}$, and the TS is symmetric, with the two forming C-C bonds having the lengths of $2.22 \AA$ (Figure 1a). Note that in the pre-reaction complex, ethylene is perpendicular to the plane of the cyclopentadiene ring. Our results are consistent with prior computational studies of this reaction. ${ }^{57,58}$ For the reactions between the more polar reactants (b and c, respectively), lower barriers are calculated: $15.5 \mathrm{kcal} / \mathrm{mol}(16.0 \mathrm{kcal} / \mathrm{mol})$ for the endo (respectively, exo) reaction of 1-methoxycyclopentadiene and cyanoethylene, and $15.8 \mathrm{kcal} / \mathrm{mol}(16.8 \mathrm{kcal} / \mathrm{mol})$ for the endo (respectively, exo) reaction of cyclopentadiene and maleic anhydride. Lower barriers are due to the electron-donating effects on the diene (reaction b) and electron withdrawing effects on the dieneophile (reactions b and c). As expected, the endo reaction is favored in both (b) and (c). For the reaction with high asymmetry and polar substituents (Figure 1b), a significant asynchronicity in seen at the TS. Note that our computed $\Delta E^{\ddagger}$ of the reaction (a) and (c) differ from those reported by Shaik since they calculated the electronic barriers in the gas phase, whereas we modeled solvation in water. ${ }^{24}$ It has been shown that the Diels-Alder reaction rate is increased in polar solvents, such as water. ${ }^{59-61}$ While there has shown to be issues with utilizing both a polarizable continuum as an implicit solvent and an external electric field, ${ }^{62}$ often times it is necessary to consider both (such as in the active site of an enzyme). Also, this current work is not focused on understand the role solvation plays in the Diels-Alder reaction with and without the presence of an exciting field. Furthermore, our results are consistent with a previous study which calculated the reaction barrier for reaction (c) endo (exo) to be 17.5 (18.8) kcal/mol with the M06-2X functional. ${ }^{63}$

The applied uniform external electric field, $\mathbf{E}_{\text {ext }}$, in the range of $\pm 20 \times 10^{-4}$ a.u., has 

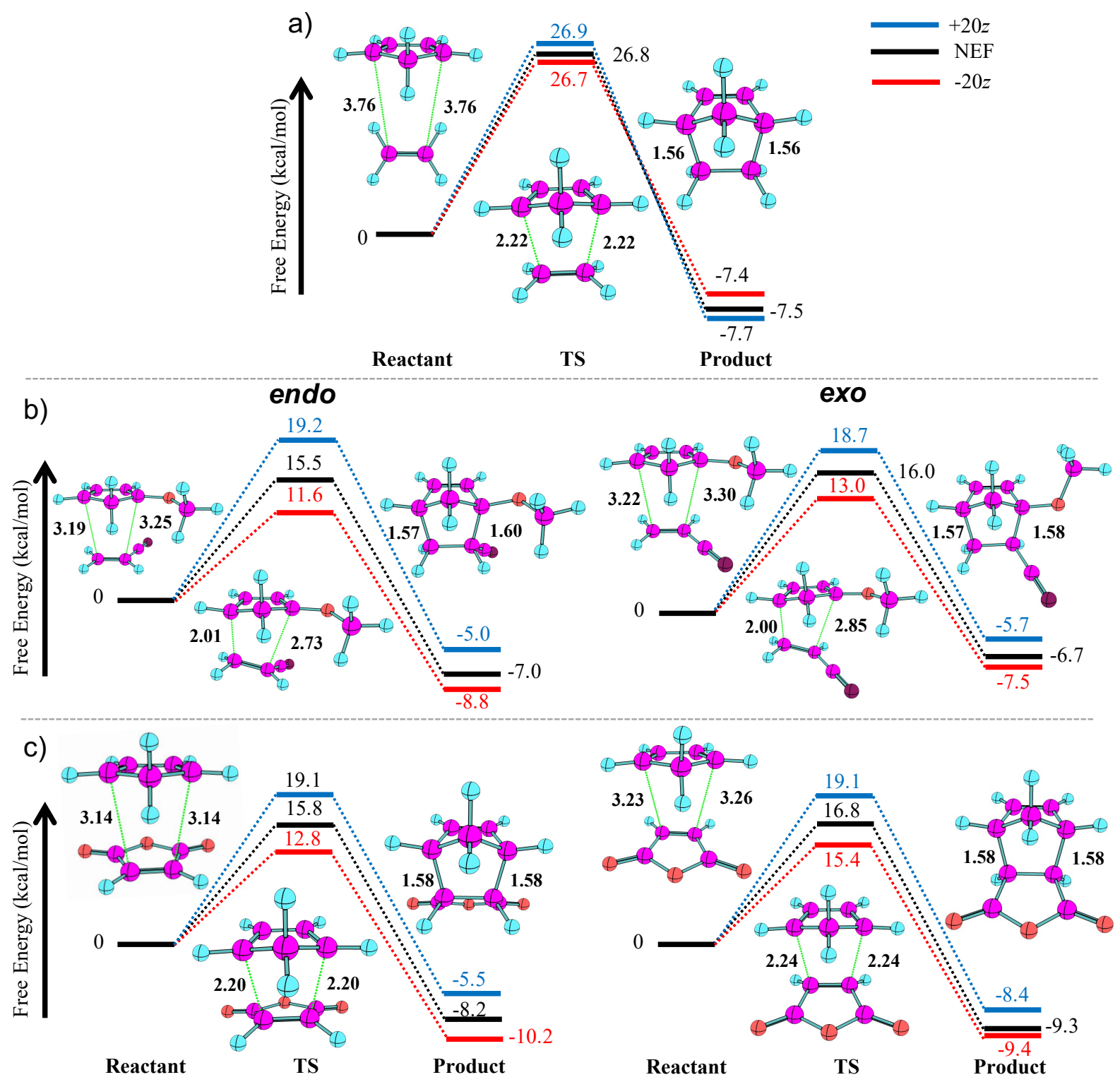

Figure 1: Calculated free energy (in kcal/mol) profiles of considered Diels-Alder reactions. Shown structures along the reaction pathway are for no external field (NEF). $\pm 20 z$ represents $\left|\mathbf{E}_{\text {ext }}\right|= \pm 20 \times 10^{-4}$ a.u. in the $z$-direction. Distances shown are in $\AA$. 
an effect on each reaction (Figures 1 and 2); the more polarity there is in the system, the greater the effect (Figure S2). For all reactions, the increase in $\mathbf{E}_{\text {ext }}$ applied in the $z$ direction increases the reaction barrier (both $\Delta G^{\ddagger}$, and $\Delta E^{\ddagger}$ ) (Figure 2). $\Delta G$ of the reaction generally increases as well, thought the non-polar reaction (a) is the exception, with $\Delta G$ slightly decreasing with the growing fields. $\mathbf{E}_{\text {ext }}$ alters the reaction barrier by affecting both the reactants, and the TS, and the TS is affected more, which is to be expected due to the increased charge transfer at the TS. ${ }^{24}$ In addition, the TS free energy and electronic energy are smooth functions of $\mathbf{E}_{\text {ext }}$, with a slightly parabolic relationship rather than strictly linear. For the reactant states, the electronic energy is smooth as a function of the field applied, whereas the free energy is not. This indicates that there is some amount of anharmanicity involved the reactant state as the field increases. This anharmanicity is likely in the motion by which the reactants come together, and hence is fixed at the TS (excluded from harmonic approximation of the free-energy) resulting in a smooth function for the TS free-energy.
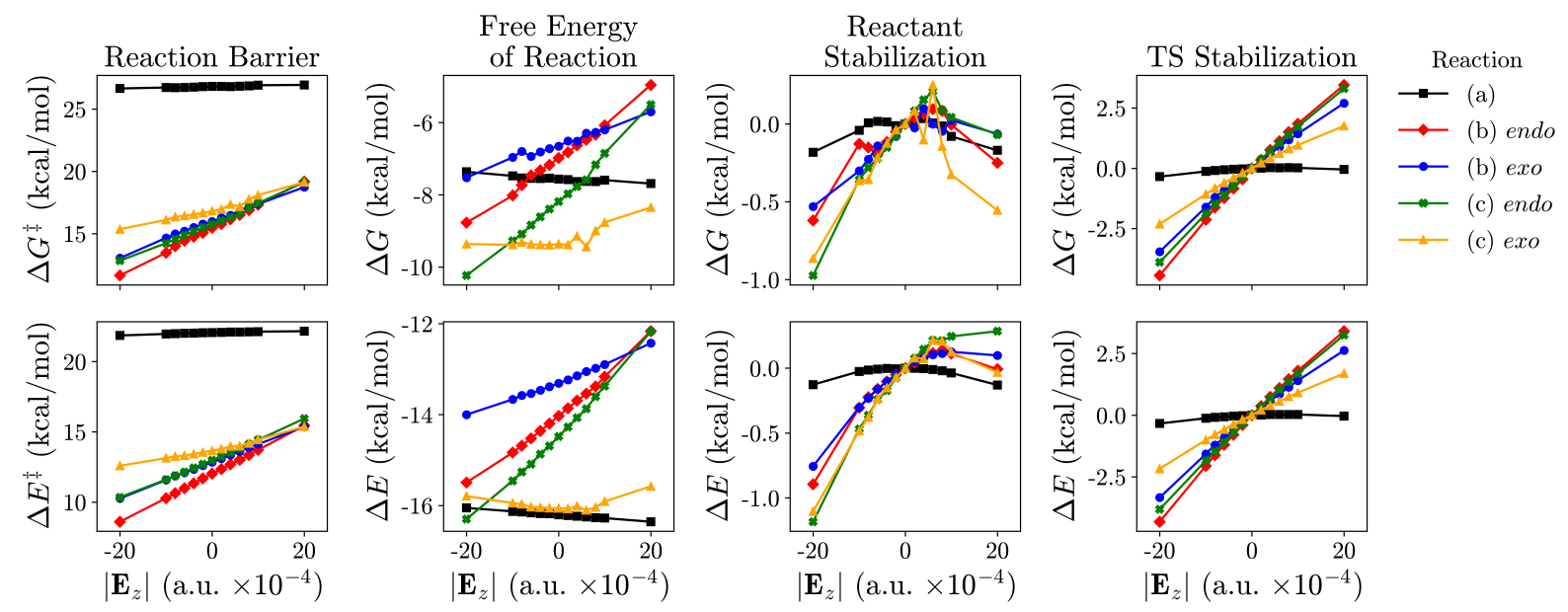

Figure 2: Reaction barrier $\left(\Delta G^{\ddagger}\right.$ and $\left.\Delta E^{\ddagger}\right)$, free energy of reaction $(\Delta G)$, reactant and TS (de)stabilization (negative values - stabilization, positive - destabilization), for all reactions as a function of $\mathbf{E}_{\text {ext }}$. For reaction (a) (black lines), a zoomed-in graph is shown in the SI to emphasize that it still responds to $\mathbf{E}_{e x t}$, despite the effect being almost negligible.

The Diels-Alder reaction is often rationalized through Frontier Molecular Orbital Theory (FMOT). ${ }^{64-66}$ Within FMOT, the highest occupied molecular orbital (HOMO) of the diene reacts with the lowest unoccupied molecular orbital (LUMO) of the dienophile to form the 
two new C-C bonds. $\mathbf{E}_{\text {ext }}$ affects the energies of both the HOMO, and the LUMO, as well as the HOMO-LUMO gap (Figure 3). In general, the LUMO responds the strongest to $\mathbf{E}_{\text {ext }}$, with a change in energy of about $\pm 7.5 \mathrm{kcal} / \mathrm{mol}$ for reactions (b) and (c), whereas the HOMO only shifts by about half of this. The HOMO-LUMO gap increases/decreases under the field applied in the positive/negative $z$-direction, leading to an increase/decrease in the barrier, respectively. The one outlier is reaction (c) exo with the HOMO energy showing a discontinuity at an applied field of $+10 \times 10^{-4}$ a.u. In fact, there is a slight, yet substantial change in the reactant state geometry for reaction (c) exo at this field. Because of the substantial change in the nuclear coordinates for fields above $+10 \times 10^{-4}$ a.u. in reaction (c) exo, we omit these data points from the correlations constructed in the rest of this study.

With the set of over 60 computed reactions in the absence of, and under various external fields, we now aim to identify the metrics of Diels-Alder reactivity in the intramolecular electric field, $\mathbf{E}(\mathbf{r})$. Often, the evaluation of $\mathbf{E}(\mathbf{r})$ at discrete points is used to analyze the response of the reaction to $\mathbf{E}_{\text {ext }}(\mathbf{r}) \cdot{ }^{17,30,67-69}$ Firstly, for a 3-D reaction, such as DielsAlder, it is not clear which point(s) to analyze. Further, we recently showed that, because $\mathbf{E}(\mathbf{r})$ is highly non-uniform, a point-based approach can produce contradictory conclusions depending on the location chosen. ${ }^{29}$ Indeed, E(r) (plotted in Figure 4 for our reaction (b) exo, for example) contains various topological features including attracting nodes, repelling nodes, and saddle points. The motion of these features is an important part of the response of $\mathbf{E}(\mathbf{r})$ to substitutions, geometric changes, and $\mathbf{E}_{\text {ext }}$. Choosing one point in association with nuclear positions may correspond to widely varying context in $\mathbf{E}(\mathbf{r})$ under even mild perturbations to the system. Therefore, a more global view on the field is necessary which accounts for the geometry of the vector field. We use a global distribution of streamlines to analyze the topology of $\mathbf{E}(\mathbf{r})$ in three regions within the Diels-Alder reactant complex: the two forming $\mathrm{C}-\mathrm{C}$ bonds, and the dienophile double bond region (Figure 4). The analysis could include more areas, or even encompass the entire complex; however, here we narrow down our exploration based on the FMOT analysis that showed that the dienophilie-based 

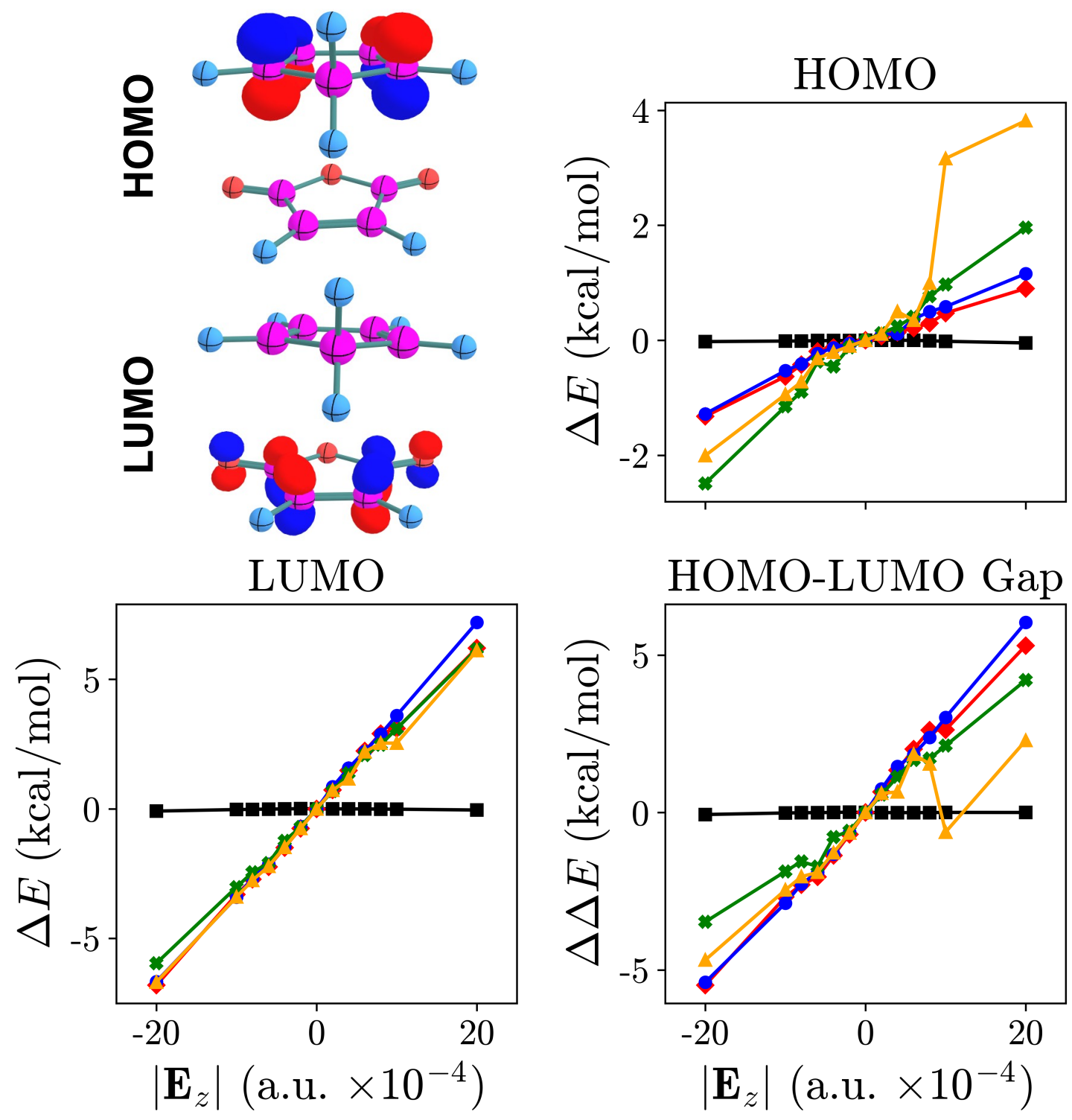

Reaction

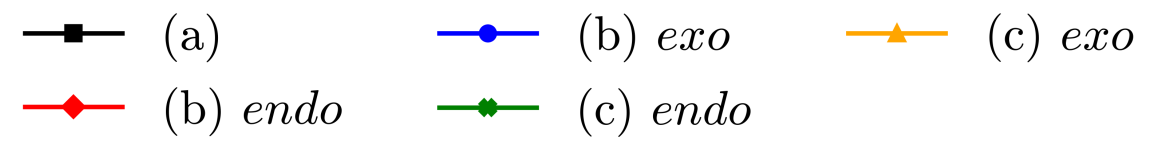

Figure 3: HOMO and LUMO for reaction (c) endo (other reactions have similar frontier orbitals). Shifts of the HOMO and LUMO energies, and of the HOMO-LUMO gap as a function of the field for each reaction, referenced to the energies at zero field. $\Delta E>0$ implies that the MO is higher in energy than the MO when no external field is applied, and $\Delta \Delta E>0$ implies that the HOMO-LUMO gap is larger than the HOMO-LUMO gap when no external field is applied. See SI for a zoomed-in graphs of reaction (a). 
LUMO responds the most to $\mathbf{E}_{\text {ext }}$, whereas the diene-based HOMO is less sensitive (Figure $3)$.
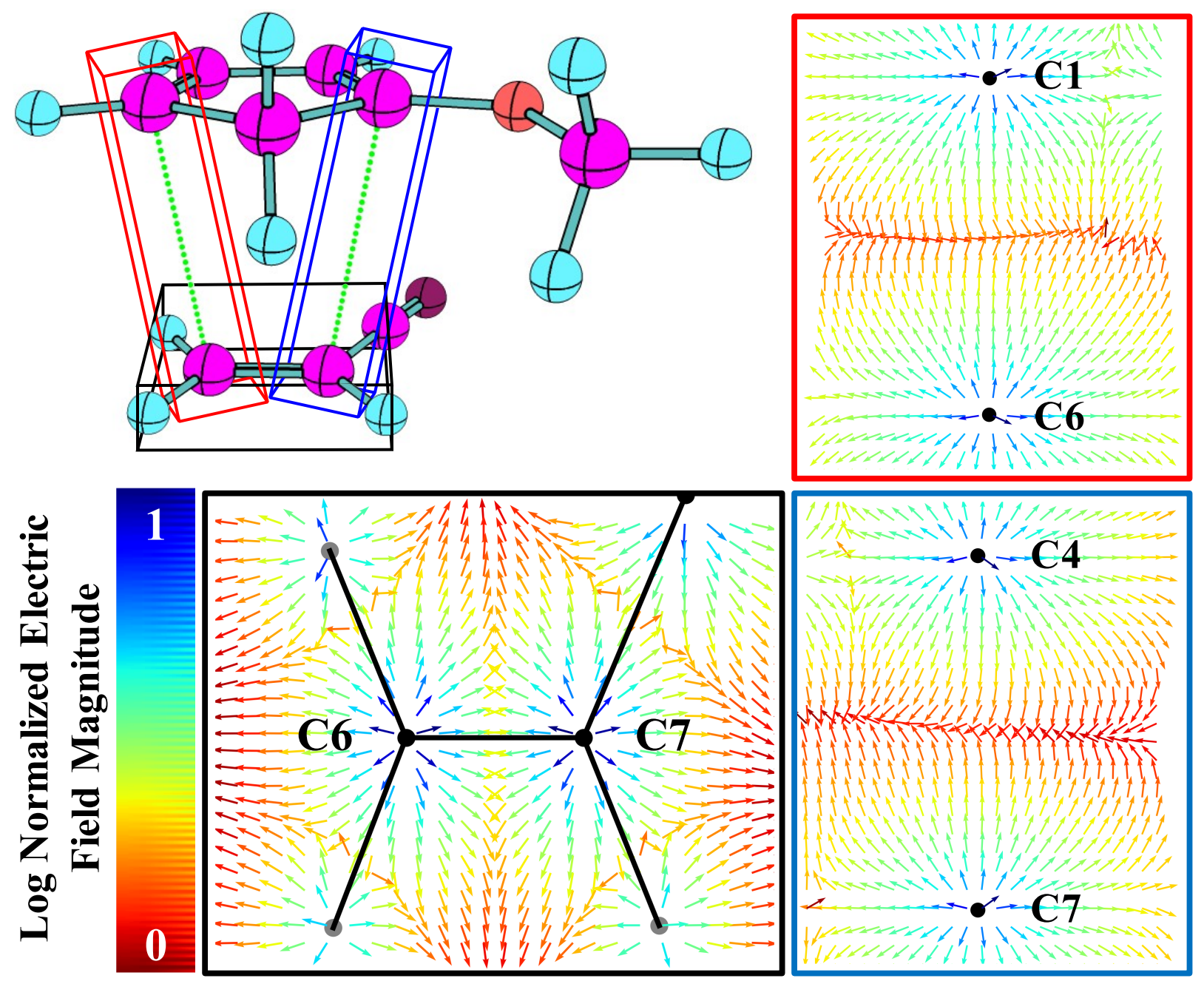

Figure 4: Locations explored in the topological analysis of $\mathbf{E}(\mathbf{r})$ without an applied external field. Red is $\mathrm{C} 1-\mathrm{C} 6$, blue is $\mathrm{C} 4-\mathrm{C} 7$, and black is $\mathrm{C6-C7}$. $|\mathbf{E}(\mathbf{r})|$ is $\log$ normalized within each region shown. Within the vector fields, black dots are carbon, gray dots are hydrogen, and black lines represent bonds.

$\mathbf{E}(\mathbf{r})$ within the volumes outlined in Figure 4 are compared across all variations of each of the three reactions. The results are summarized in the dissimilarity matrices (Figure 5, left column for the C6-C7 region, and Figures S12-S16 in the Supporting Information for the rest). The diagonal in a matrix is always black, representing a comparison of a field topology to itself. Off-diagonal terms can range from black to white, with lighter colors 
signifying greater differences in electric field topologies. The matrix for reaction (a) behaves most predictably: the greater the difference in the applied electric field, the more different is the field within the C6-C7 region. Reaction (b) endo also shows this for smaller fields (the center portion of the matrix); however, for larger fields, we see an onset of differences of the topology of $\mathbf{E}(\mathbf{r})$. For reaction (c) exo, we see the largest jump in the topologies at +10 and +20 ; recall that this system showed the discontinuity of the HOMO energy at these fields (Figure 3), and substantial changes in the nuclear coordinates. Overall, for all reactions, there transpires a correlation between the magnitudes of the applied fields and how similar the topologies of the intramolecular field are. Since we already saw that the reaction barrier responds to the external field as a smooth function (Figure 2), we can expect that the field topology similarity would report on the similarity of the barriers.

Indeed, the difference in reaction barrier $\left(\Delta \Delta G_{f, g}^{\ddagger}\right)$ and the dissimilarity in the electric field topology $(D(f, g))$ are correlated, and show an exponential relationship (Figure 5, right column, and Figure S13-S17 in the Supporting Information). In general, we see good correlations between the electric field topology and reaction barrier for all volumes analyzed (Figure 4) and all reactions (Table 1). Interestingly, for reaction (c) exo, two separate correlations emerge as a result of the large change in the HOMO energy (Figure 3), and the red "x" points correspond to the white regions in the matrices and a geometric change due to $\mathbf{E}_{\text {ext }}$. These red points are excluded from the regression analysis.

Table 1: $\quad R^{2}$ values for the electric field regions analyzed (Figure 4) fit to an exponential curve.

\begin{tabular}{|c|c|c|c|c|c|}
\hline Region & (a) & (b) endo & (b) exo & (c) endo & (c) exo \\
\hline$c 1-c 6$ & 0.59 & 0.61 & 0.55 & 0.54 & 0.50 \\
\hline$c 4-c 7$ & 0.65 & 0.62 & 0.54 & 0.57 & 0.47 \\
\hline$c 6-c 7$ & 0.64 & 0.68 & 0.73 & 0.64 & 0.57 \\
\hline
\end{tabular}

Since intramolecular fields in the reactant state contain features that are descriptive and/or predictive of the subsequent Diels-Alder reactivity, there should be topological features of $\rho(\mathbf{r})$, in correspondence with those in $\mathbf{E}(\mathbf{r})$, that also correlate with this reactivity. 

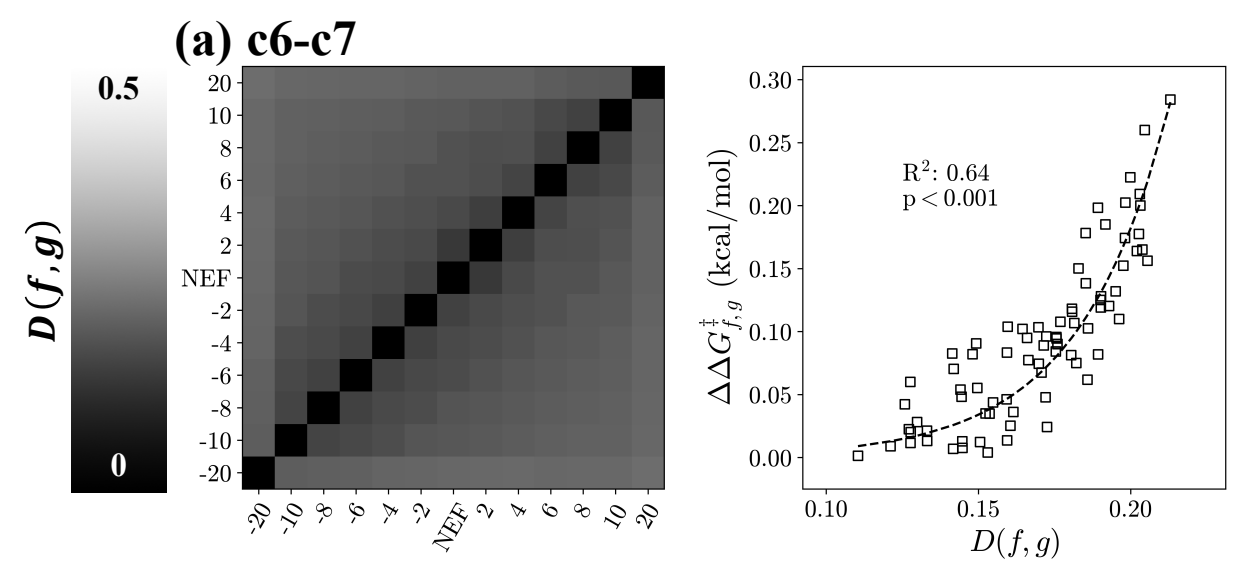

(b) endo c6-c7
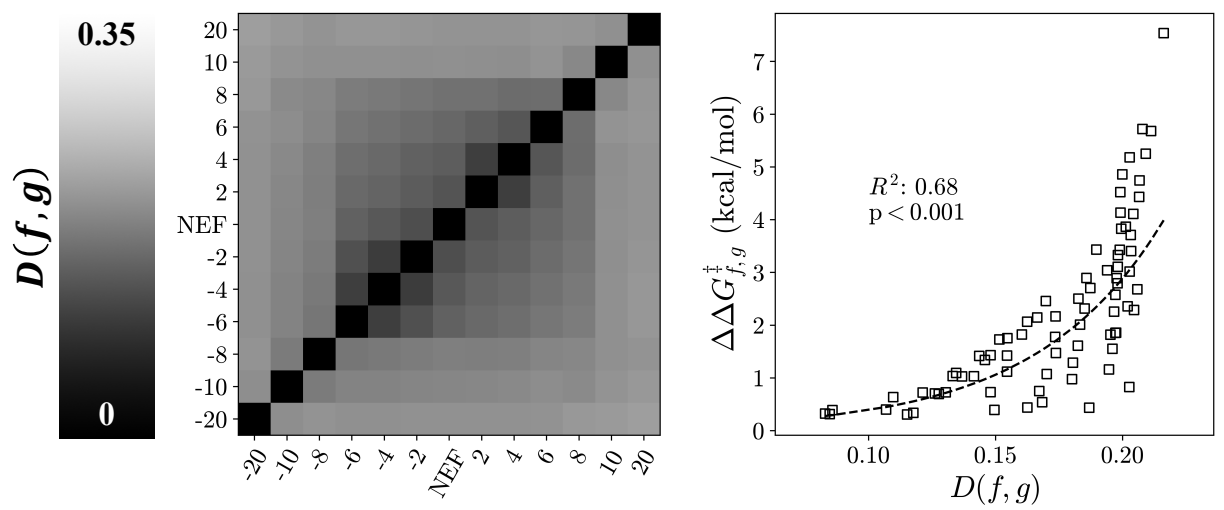

\section{(c) exo c6-c7}
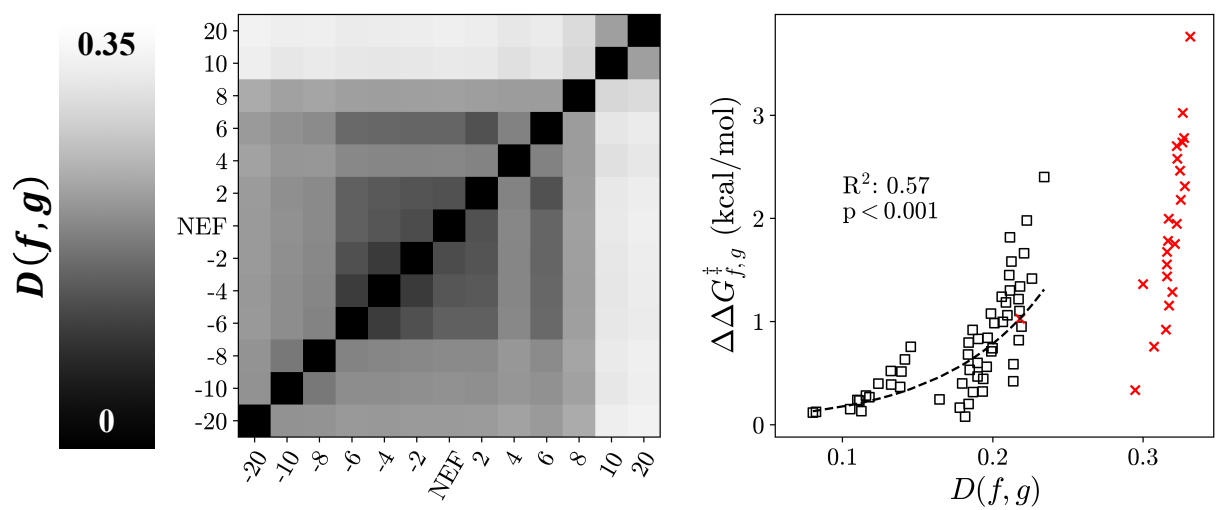

Figure 5: (Left) Dissimilarity matrices between the field topologies within the C6-C7 volume. Numbers represent magnitude of applied field $\times 10^{-4}$ a.u.; NEF stands for no external field; black: the same topology, white: the greatest topology difference. (Right) The dissimilarities of the field topology $(D(f, g))$ plotted against the reaction barrier difference $\left(\Delta \Delta G_{f, g}^{\ddagger}\right)$, with the corresponding $R^{2}$ and $p$ values. Red "x" symbols correspond to the fields at which reaction (c) exo showed a geometric change and a discontinuity in orbital energies. 
Using QTAIM, we zoom onto the RCP of the cyclopentadiene, and the BCP of the dieneophile double bond (Figure 6). The promising locations are picked on the basis of all the findings so far. A broader set of computed QTAIM parameters is given in the Supporting Information. The correlations between each QTAIM parameter and $\Delta G^{\ddagger}$ of the reaction, represented as $R^{2}$ values, are summarized in Figure 6 (Bottom), and Tables 2 and $\mathrm{S} 1$ in the Supporting Information.
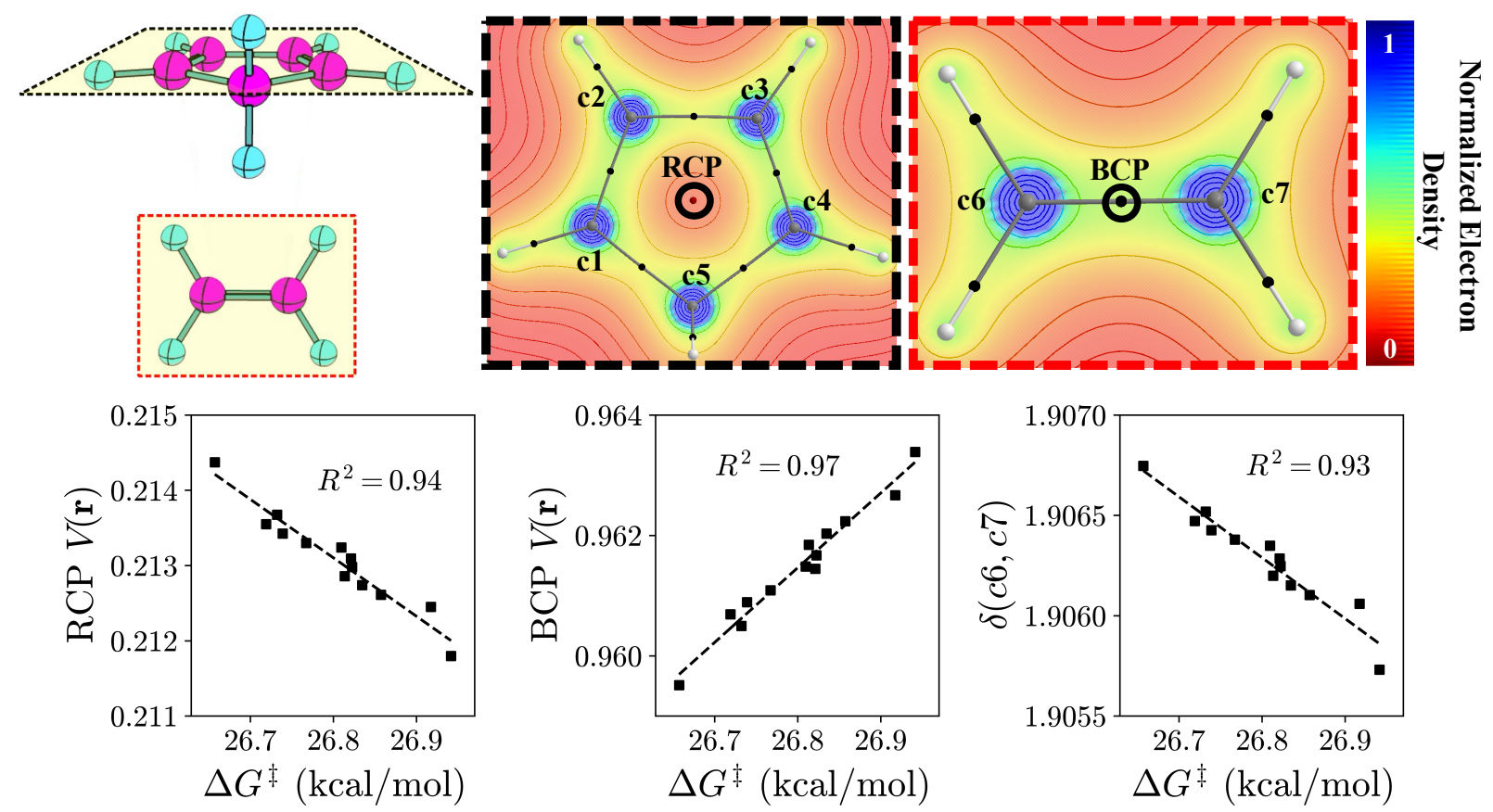

Figure 6: Location used for the QTAIM analysis. (Top) For the structure at left, the slices $\rho(\mathbf{r})$ along the indicated planes are shown. The RCP in the cyclopentadiene ring, and the BCP lies in the dieneophile double bond are indicated. (Bottom) Correlation between QTAIM values and the reaction barrier for reaction (a).

For all reactions, $V\left(\mathbf{r}_{\mathbf{c}}\right)$ at the $\mathrm{BCP}$ and $\mathrm{RCP}$, and $\delta(C 6, C 7)$ correlate strongest with the reaction barrier $\left(R^{2} \geq 0.90\right)$. All other metrics correlate strongly with the barrier of reactions (b) and (c); however, correlate weakly with the barrier of reaction (a). We note that reaction (a) barely responded to the external electric field (shift of $0.1 \mathrm{kcal} / \mathrm{mol}$ in the barrier, Figure 2), and similarly, changes in the various topological parameters of $\rho\left(\mathbf{r}_{\mathbf{c}}\right)$ are quite small (Figure 6 and Figure S5 in the Supporting Information). Furthermore, for 
Table 2: $R^{2}$ values for the corresponding QTAIM parameter to reaction barrier $\left(\Delta G^{\ddagger}\right)$. RCP and $\mathrm{BCP}$ are identified in Figure 6. Atoms $C 6$ and $C 7$ are defined in Scheme 1 and Figure 6.

\begin{tabular}{|l|c|c|c|c|c|}
\hline QTAIM Parameter & $(\mathrm{a})$ & $(\mathrm{b})$ endo & $(\mathrm{b})$ exo & (c) endo & $(\mathrm{c})$ exo \\
\hline $\mathrm{RCP} \rho\left(\mathbf{r}_{\mathbf{c}}\right)$ & 0.41 & 0.82 & 0.93 & 0.96 & 0.96 \\
\hline $\mathrm{RCP} \nabla^{2} \rho\left(\mathbf{r}_{\mathbf{c}}\right)$ & 0.26 & 0.91 & 0.95 & 0.98 & 0.97 \\
\hline $\operatorname{RCP} V\left(\mathbf{r}_{\mathbf{c}}\right)$ & 0.94 & 0.97 & 0.99 & 0.98 & 0.97 \\
\hline $\operatorname{RCP} \mathcal{G}\left(\mathbf{r}_{\mathbf{c}}\right)$ & 0.28 & 0.93 & 0.95 & 0.97 & 0.97 \\
\hline $\operatorname{BCP} \rho\left(\mathbf{r}_{\mathbf{c}}\right)$ & 0.44 & 0.95 & 0.97 & 0.96 & 0.94 \\
\hline $\operatorname{BCP} \nabla^{2} \rho\left(\mathbf{r}_{\mathbf{c}}\right)$ & 0.44 & 0.96 & 0.97 & 0.96 & 0.94 \\
\hline $\operatorname{BCP} V\left(\mathbf{r}_{\mathbf{c}}\right)$ & 0.97 & 0.99 & 1.00 & 0.98 & 0.97 \\
\hline $\operatorname{BCP} \mathcal{G}\left(\mathbf{r}_{\mathbf{c}}\right)$ & 0.46 & 0.91 & 0.96 & 0.95 & 0.95 \\
\hline$\delta(C 6, C 7)$ & 0.93 & 0.96 & 0.98 & 0.95 & 0.95 \\
\hline
\end{tabular}

reaction (a), an increased barrier corresponds to a lower $\delta(C 6, C 7)$, which is indicative of a preemptive breaking of the dienophile double bond. One would expect this to cause a lower barrier, and indeed, for the polar reactions (b) and (c), we see a lower $\delta(C 6, C 7)$ corresponding to a lower barrier (See Figure S6 and S7 in the Supporting Information). This discrepancy for reaction (a) is likely due to the orthogonal orientation of ethylene with respect to the cyclopentadiene ring, different from the other two reactions. Both $\mathcal{G}\left(\mathbf{r}_{\mathbf{c}}\right)$ and $\nabla^{2} \rho\left(\mathbf{r}_{\mathbf{c}}\right)$ correlate with $\Delta G^{\ddagger}$, especially for larger changes in $\Delta G^{\ddagger}$, as for reaction (b) and (c). Indeed, $\mathcal{G}\left(\mathbf{r}_{\mathbf{c}}\right)$ and $\nabla^{2} \rho\left(\mathbf{r}_{\mathbf{c}}\right)$ are often used as measures of the energy within a bond or ring, and the weaker the double bond in the dieneophile, the lower $\Delta G^{\ddagger}$.

For reactions involving $\mathbf{E}_{e x t}, V\left(\mathbf{r}_{\mathbf{c}}\right)$ correlates the strongest with $\Delta G^{\ddagger}$. That indeed makes sense: when we apply an external field of magnitude $\mathbf{E}_{\text {ext }}$ to the system, $\rho(\mathbf{r})$ should experience a force density $\mathbf{f}(\mathbf{r})=\rho(\mathbf{r}) \mathbf{E}_{\text {ext }}$. From classical electrodynamics, $V(\mathbf{r})$ can be calculated directly from $\mathbf{E}_{\text {int }}(\mathbf{r})$ (which depends on $\rho(\mathbf{r})$ ), see Equation 1) using Equation 5, where $\mathcal{O}$ is taken to be our reference point.

$$
V(\mathbf{r})=-\int_{\mathcal{O}}^{\mathbf{r}} \mathbf{E}_{\text {int }} \cdot d \mathbf{l}
$$

However, if we assume $\rho(\mathbf{r}) \rightarrow 0$ as $\mathbf{r} \rightarrow \infty$ (which should generally hold true for molecules), 
we can instead calculate the potential from Equation 6.

$$
V(\mathbf{r})=-\int d \tau^{\prime} \frac{\rho\left(\mathbf{r}^{\prime}\right)}{\left|\mathbf{r}-\mathbf{r}^{\prime}\right|}+\sum_{i} \frac{Z_{i}}{\left|\mathbf{r}-\mathbf{R}_{i}\right|}
$$

Note that Equation 6 omits the contribution from $\mathbf{E}_{\text {ext }}$ as QTAIM does not include the external field when it calculates $V(\mathbf{r})$. Furthermore, if we wanted to include $\mathbf{E}_{\text {ext }}$, we could not use Equation 6 as it assumes the reference point is taken to be $\infty$, and the line integral (Equation 5) does not converge if $\mathcal{O}=\infty$. Hence, we can expect that $V(\mathbf{r})$ should correlate with the reaction barrier, which is dependent on the external field we apply to the system.

In general, the QTAIM parameters presented in Table 2 can be valuable metrics for detecting changes in the reaction barrier from within the reactant state. This supports our findings that $\mathbf{E}_{\text {ext }}$ alters the reactivity mainly by affecting the dienophile bond. We additionally see that the number of delocalized electrons in that bond $(\delta(C 6, C 7))$ correlates strongly with $\Delta G^{\ddagger}$, again confirming our findings. Overall, the double bond of the dienophile appears to be the focal point of affecting the Diels-Alder reactivity. We conclude that the most effective way to speed up the Diels-Alder via external fields is by applying a field that weakens the double bond on the dienophile in the reactant state. This can be achieved most effectively for reaction (b) and (c), and other Diels-Alder reactions with polar substituents or $\pi$-resonance opportunities in the dienophile.

\section{Conclusion}

External electric fields have the ability to control and alter the reaction rate of various chemical reactions and can be an indispensable tool in catalysis. However, when the reaction of interest involves multiple bonds forming/shifting in 3-D, and more than one dipole created/relocated, as are many organic reactions, choosing the direction or the field for catalysis, or even deciding whether the reaction can be field-catalyzed becomes non-trivial. Previous studies, both computational and experimental, have shown how an external field 
can alter the reactivity and selectivity of a reaction; however, none have localized the extent of the effect on a particular fragment. ${ }^{70-72}$ In particular, it is of interest to understand what local properties in the system correlate to changes in the external field as well as the reaction barrier as an intermediate computational and experimental probe for understanding the extent of an otherwise non-uniform external field. While there already exists reactivity descriptors for the Diels-Alder reaction (FMOT among others), these metrics are specific exclusively to the Diels-Alder reaction and do not necessarily translate to other reactions. We propose an approach, which should be transferable to all sorts of reactions, to detect and predict how a reaction rate would respond to an external field. Our starting point is the intramolecular field native to the reactant complex, to which an external field (of a realistic magnitude, up to $10 \mathrm{MV} / \mathrm{cm}$ ) can be applied as a relatively mild perturbation. Note the discussed intramolecular fields, created by all nuclei, electrons, and the external component, are highly-heterogeneous, and cannot be viewed as a simple single vector. We analyze and rigorously compare the global 3-dimensional topologies of these intramolecular electric fields. We show that features in the global topology of the intramolecular fields can be identified such that they report on the free energy barrier of the reaction to follow. Specifically, we show this on the example of the Diels-Alder reaction, one of the most widely studied reactions, indeed. The most direct measure of the external field effect on the reaction appears to be $\mathbf{E}(\mathbf{r})$ in the volume surrounding the double bond of the dienophile. Interestingly, the topological similarity of $\mathbf{E}(\mathbf{r})$ across different versions of the Diels-Alder reaction is related exponentially to $\Delta \Delta G^{\ddagger}$, with the fundamental source of this dependence to be further investigated. In geometric correspondence with the most descriptive location for $\mathbf{E}(\mathbf{r})$ in the reactant complex, features in the topology of $\rho(\mathbf{r})$ are found, which are also predictive of the barrier. The reason for this is that the field both is affected by electron density, and affects it, with implications for reactivity. Experimentally, our results indicate that Linear Stark Spectroscopy aimed at evaluating the electric field on the dienophile bond in the Diels-Alder reaction is the most promising for predicting changes in the reaction barrier. For computation, being 
able to predict the barrier from the reactant state without a mechanistic calculation is a welcome simplification. In addition, since the topology of intramolecular fields is a metric of reactivity, its features can constitute a rigorous and physically grounded set of descriptors for machine learning. Finally, we believe that our findings and the methodology will enable the design of most effective external field for catalysis of this, and many other reactions, which potentially could be realized in such molecular frameworks as an enzyme or zeolite structure.

\section{Supporting Information Available}

Enhanced graphs for reaction (a), 2D histograms of curvature versus Euclidean distance, plots of QTAIM correlations, additional QTAIM correlations computed, schematic of various

topological features in $\rho(\mathbf{r})$ and $\mathbf{E}(\mathbf{r}), \Delta \Delta G_{f, g}^{\ddagger}$ versus $D(f, g)$ for all reactions, and structure coordinates for the reaction with no external field applied.

\section{Acknowledgement}

This work was supported by the National Science Foundation grant CHE-1903808 and used the Extreme Science and Engineering Discovery Environment (XSEDE) Bridges system at the Pittsburgh Supercomputing Center (PSC). We also acknowledge the UCLA-IDRE cluster Hoffman2 computational resources.

\section{References}

(1) Shaik, S.; de Visser, S. P.; Kumar, D. External Electric Field Will Control the Selectivity of Enzymatic-Like Bond Activations. J. Am. Chem. Soc. 2004, 126, 11746-11749.

(2) Warshel, A. Electrostatic Origin of the Catalytic Power of Enzymes and the Role of Preorganized Active Sites. J. Biol. Chem. 1998, 273, 27035-27038. 
(3) Fried, S. D.; Bagchi, S.; Boxer, S. G. Extreme electric fields power catalysis in the active site of ketosteroid isomerase. Science 2014, 346, 1510-1514.

(4) Pacchioni, G.; Lomas, J. R.; Illas, F. Electric field effects in heterogeneous catalysis. J. Mol. Catal. A: Chem. 1997, 119, 263-273.

(5) Lee, V.-J. Heterogeneous Catalysis: Effect of an Alternating Electric Field. Science 1966, 152, 514-514.

(6) Simons, R. Strong electric field effects on proton transfer between membrane-bound amines and water. Nature 1979, 280, 824-826.

(7) Mansoor, E.; Van der Mynsbrugge, J.; Head-Gordon, M.; Bell, A. T. Impact of longrange electrostatic and dispersive interactions on theoretical predictions of adsorption and catalysis in zeolites. Catal. Today 2018, 312, 51-65.

(8) Shaik, S.; Mandal, D.; Ramanan, R. Oriented electric fields as future smart reagents in chemistry. Nat. Chem. 2016, 8, 1091-1098.

(9) Warshel, A.; Sharma, P. K.; Kato, M.; Xiang, Y.; Liu, H.; Olsson, M. H. M. Electrostatic Basis for Enzyme Catalysis. Chem. Rev. 2006, 106, 3210-3235.

(10) Fried, S. D.; Boxer, S. G. Electric Fields and Enzyme Catalysis. Annu. Rev. Biochem. 2017, 86, 387-415.

(11) Warshel, A.; Sharma, P. K.; Chu, Z. T.; Åqvist, J. Electrostatic Contributions to Binding of Transition State Analogues Can Be Very Different from the Corresponding Contributions to Catalysis: Phenolates Binding to the Oxyanion Hole of Ketosteroid Isomerase †. Biochemistry 2007, 46, 1466-1476.

(12) Fuller III, J.; Wilson, T. R.; Eberhart, M. E.; Alexandrova, A. N.; Fuller, J.; Wilson, T. R.; Eberhart, M. E.; Alexandrova, A. N. Charge Density in Enzyme Active 
Site as a Descriptor of Electrostatic Preorganization. J. Chem. Inf. Model. 2019, 59, 2367-2373.

(13) Layfield, J. P.; Hammes-Schiffer, S. Calculation of Vibrational Shifts of Nitrile Probes in the Active Site of Ketosteroid Isomerase upon Ligand Binding. J. Am. Chem. Soc. 2013, 135, 717-725.

(14) Kamerlin, S. C. L.; Sharma, P. K.; Chu, Z. T.; Warshel, A. Ketosteroid isomerase provides further support for the idea that enzymes work by electrostatic preorganization. Proc. Natl. Acad. Sci. U.S.A. 2010, 10\%, 4075-4080.

(15) Park, H.; Merz, K. M. Molecular Dynamics and Quantum Chemical Studies on the Catalytic Mechanism of $\Delta 5$-3-Ketosteroid Isomerase: The Catalytic Diad versus the Cooperative Hydrogen Bond Mechanism. J. Am. Chem. Soc. 2003, 125, 901-911.

(16) Houck, W. J.; Pollack, R. M. Temperature Effects on the Catalytic Activity of the D38E Mutant of 3-Oxo- $\Delta 5$-Steroid Isomerase: Favorable Enthalpies and Entropies of Activation Relative to the Nonenzymatic Reaction Catalyzed by Acetate Ion. J. Am. Chem. Soc. 2004, 126, 16416-16425.

(17) Kȩdzierski, P.; Zaczkowska, M.; Sokalski, W. A. Extreme Catalytic Power of Ketosteroid Isomerase Related to the Reversal of Proton Dislocations in Hydrogen-Bond Network. J. Phys. Chem. B 2020, 124, 3661-3666.

(18) Kraut, D. A.; Sigala, P. A.; Pybus, B.; Liu, C. W.; Ringe, D.; Petsko, G. A.; Herschlag, D. Testing Electrostatic Complementarity in Enzyme Catalysis: Hydrogen Bonding in the Ketosteroid Isomerase Oxyanion Hole. PLoS Biol. 2006, 4, e99.

(19) Herschlag, D.; Natarajan, A. Fundamental Challenges in Mechanistic Enzymology: Progress toward Understanding the Rate Enhancements of Enzymes. Biochemistry 2013, 52, 2050-2067. 
(20) Wu, Y.; Boxer, S. G. A critical test of the electrostatic contribution to catalysis with noncanonical Amino Acids in ketosteroid isomerase. J. Am. Chem. Soc. 2016, 138, $11890-11895$.

(21) Wu, Y.; Fried, S. D.; Boxer, S. G. A Preorganized Electric Field Leads to Minimal Geometrical Reorientation in the Catalytic Reaction of Ketosteroid Isomerase. J. Am. Chem. Soc. 2020, 142, 9993-9998.

(22) Wang, Z.; Danovich, D.; Ramanan, R.; Shaik, S. Oriented-External Electric Fields Create Absolute Enantioselectivity in Diels-Alder Reactions: Importance of the Molecular Dipole Moment. J. Am. Chem. Soc. 2018, 140, 13350-13359.

(23) Timerghazin, Q. K.; Talipov, M. R. Unprecedented External Electric Field Effects on S -Nitrosothiols: Possible Mechanism of Biological Regulation? J. Phys. Chem. Lett. 2013, 4, 1034-1038.

(24) Meir, R.; Chen, H.; Lai, W.; Shaik, S. Oriented electric fields accelerate diels-alder reactions and control the endo/exo selectivity. ChemPhysChem 2010, 11, 301-310.

(25) Jissy, A. K.; Datta, A. Effect of External Electric Field on H-Bonding and $\pi$-Stacking Interactions in Guanine Aggregates. ChemPhysChem 2012, 13, 4163-4172.

(26) Aitken, H. M.; Coote, M. L. Can electrostatic catalysis of Diels-Alder reactions be harnessed with pH-switchable charged functional groups? Phys. Chem. Chem. Phys. 2018, 20, 10671-10676.

(27) Bhattacharyya, K.; Karmakar, S.; Datta, A. External electric field control: driving the reactivity of metal-free azide-alkyne click reactions. Phys. Chem. Chem. Phys. 2017, 19, 22482-22486.

(28) Aragonès, A. C.; Haworth, N. L.; Darwish, N.; Ciampi, S.; Bloomfield, N. J.; Wal- 
lace, G. G.; Diez-Perez, I.; Coote, M. L. Electrostatic catalysis of a Diels-Alder reaction. Nature 2016, 531, 88-91.

(29) Hennefarth, M. R.; Alexandrova, A. N. Direct Look at the Electric Field in Ketosteroid Isomerase and Its Variants. ACS Catal. 2020, 10, 9915-9924.

(30) Wang, L.; Fried, S. D.; Markland, T. E. Proton network flexibility enables robustness and large electric fields in the ketosteroid isomerase active site. J. Phys. Chem. B 2017, 121, 9807-9815.

(31) of Karlsruhe, U.; GmbH, F. K. TURBOMOLE. http://www.turbomole.com.

(32) Ahlrichs, R.; Bär, M.; Häser, M.; Horn, H.; Kölmel, C. Electronic structure calculations on workstation computers: The program system turbomole. Chem. Phys. Lett. 1989, 162, 165-169.

(33) von Arnim, M.; Ahlrichs, R. Performance of parallel TURBOMOLE for density functional calculations. J. Comput. Chem. 1998, 19, 1746-1757.

(34) von Arnim, M.; Ahlrichs, R. Geometry optimization in generalized natural internal coordinates. The J. Chem. Phys. 1999, 111, 9183-9190.

(35) Ahlrichs, R. Efficient evaluation of three-center two-electron integrals over Gaussian functions. Phys. Chem. Chem. Phys. 2004, 6, 5119.

(36) Häser, M.; Ahlrichs, R. Improvements on the direct SCF method. J. Comput. Chem. 1989, 10, 104-111.

(37) Sierka, M.; Hogekamp, A.; Ahlrichs, R. Fast evaluation of the Coulomb potential for electron densities using multipole accelerated resolution of identity approximation. The J. Chem. Phys. 2003, 118, 9136-9148.

(38) Treutler, O.; Ahlrichs, R. Efficient molecular numerical integration schemes. The J. Chem. Phys. 1995, 102, 346-354. 
(39) Eichkorn, K.; Treutler, O.; Öhm, H.; Häser, M.; Ahlrichs, R. Auxiliary basis sets to approximate Coulomb potentials. Chem. Phys. Lett. 1995, 240, 283-290.

(40) Eichkorn, K.; Weigend, F.; Treutler, O.; Ahlrichs, R. Auxiliary basis sets for main row atoms and transition metals and their use to approximate Coulomb potentials. Theor. Chem. Acc.: Theory, Computation, and Modeling (Theor. Chim. Acta) 1997, $97,119-124$.

(41) Deglmann, P.; May, K.; Furche, F.; Ahlrichs, R. Nuclear second analytical derivative calculations using auxiliary basis set expansions. Chem. Phys. Lett. 2004, 384, 103-107.

(42) Becke, A. D. Density-functional thermochemistry. III. The role of exact exchange. The J. Chem. Phys. 1993, 98, 5648-5652.

(43) Lee, C.; Yang, W.; Parr, R. G. Development of the Colle-Salvetti correlation-energy formula into a functional of the electron density. Phys. Rev. B 1988, 37, 785-789.

(44) Vosko, S. H.; Wilk, L.; Nusair, M. Accurate spin-dependent electron liquid correlation energies for local spin density calculations: a critical analysis. Can. J. Phys. 1980, 58, $1200-1211$.

(45) Stephens, P. J.; Devlin, F. J.; Chabalowski, C. F.; Frisch, M. J. Ab Initio Calculation of Vibrational Absorption and Circular Dichroism Spectra Using Density Functional Force Fields. J. Phys. Chem. 1994, 98, 11623-11627.

(46) Weigend, F.; Ahlrichs, R. Balanced basis sets of split valence, triple zeta valence and quadruple zeta valence quality for H to Rn: Design and assessment of accuracy. Phys. Chem. Chem. Phys. 2005, 7, 3297.

(47) Ess, D. H.; Jones, G. O.; Houk, K. N. Conceptual, Qualitative, and Quantitative Theories of 1,3-Dipolar and Diels-Alder Cycloadditions Used in Synthesis. Adv. Synth. Catal. 2006, 348, 2337-2361. 
(48) Weigend, F.; Ahlrichs, R. Balanced basis sets of split valence, triple zeta valence and quadruple zeta valence quality for H to Rn: Design and assessment of accuracy. Phys. Chem. Chem. Phys. 2005, 7, 3297.

(49) Klamt, A.; Schüürmann, G. COSMO: a new approach to dielectric screening in solvents with explicit expressions for the screening energy and its gradient. J. Chem. Soc., Perkin Trans. 2 1993, 0, 799-805.

(50) Dinh, H. Q.; Xu, L. Lect. Notes Comput. Sci. (including subseries Lecture Notes in Artif. Intell. and Lecture Notes in Bioinformatics); Springer, Berlin, Heidelberg, 2008; Vol. 5342 LNCS; pp 187-196.

(51) Keith, T. A. AIMALL, TK Gristmill Software. Overland Park KS, USA,(aim. tkgristmill. com) 2016,

(52) Bader, R. F. W. Atoms in molecules. Acc. Chem. Res. 1985, 18, 9-15.

(53) Bader, R. F. W. Quantum topology of molecular charge distributions. III. The mechanics of an atom in a molecule. The J. Chem. Phys. 1980, 73, 2871-2883.

(54) Biegler-könig, F. W.; Bader, R. F. W.; Tang, T.-H. Calculation of the average properties of atoms in molecules. II. J. Comput. Chem. 1982, 3, 317-328.

(55) Bader, R. F. W. The Quantum Theory of Atoms in Molecules. Clarendon: Oxford, UK 2007

(56) Wang, Y.-G.; Werstiuk, N. H. A practical and efficient method to calculate AIM localization and delocalization indices at post-HF levels of theory. J. Comput. Chem. 2003, $24,379-385$.

(57) Levandowski, B. J.; Houk, K. Theoretical analysis of reactivity patterns in Diels-Alder reactions of cyclopentadiene, cyclohexadiene, and cycloheptadiene with symmetrical and unsymmetrical dienophiles. J. Org. Chem. 2015, 80, 3530-3537. 
(58) Black, K.; Liu, P.; Xu, L.; Doubleday, C.; Houk, K. N. Dynamics, transition states, and timing of bond formation in Diels-Alder reactions. Proc. Natl. Acad. Sci. U.S.A. 2012, 109, 12860-12865.

(59) Ruiz-Löpez, M. F.; Assfeld, X.; Garíca, J. I.; Mayoral, J. A.; Salvatella, L. Solvent Effects on the Mechanism and Selectivities of Asymmetric Diels-Alder Reactions. J. Am. Chem. Soc. 1993, 115, 8780-8787.

(60) Thomas, L. L.; Tirado-Rives, J.; Jorgensen, W. L. Quantum mechanical/molecular mechanical modeling finds diels alder reactions are accelerated less on the surface of water than in water. J. Am. Chem. Soc. 2010, 132, 3097-3104.

(61) Rideout, D. C.; Breslow, R. Hydrophobic acceleration of Diels-Alder reactions. J. Am. Chem. Soc. 1980, 102, 7816-7817.

(62) Dubey, K. D.; Stuyver, T.; Kalita, S.; Shaik, S. Solvent-Organization and RateRegulation of a Menshutkin Reaction by Oriented-External Electric Fields are Revealed by Combined MD and QM/MM Calculations. J. Am. Chem. Soc. 2020,

(63) Sakata, K.; Fujimoto, H. Origin of the endo Selectivity in the Diels-Alder Reaction between Cyclopentadiene and Maleic Anhydride. Eur. J. Org. Chem. 2016, 2016, 42754278.

(64) Houk, K. Generalized frontier orbitals of alkenes and dienes. Regioselectivity in DielsAlder reactions. J. Am. Chem. Soc. 1973, 95, 4092-4094.

(65) Houk, K. N. Frontier molecular orbital theory of cycloaddition reactions. Acc. Chem. Res. 1975, 8, 361-369.

(66) Rozeboom, M. D.; Tegmo-Larsson, I. M.; Houk, K. Frontier molecular orbital theory of substitutent effects on regioselectivities of nucleophilic additions and cycloadditions to benzoquinones and naphthoquinones. J. Org. Chem. 1981, 46, 2338-2345. 
(67) Welborn, V. V.; Head-Gordon, T. Fluctuations of Electric Fields in the Active Site of the Enzyme Ketosteroid Isomerase. J. Am. Chem. Soc. 2019, 141, 12487-12492.

(68) Wang, X.; He, X.; Zhang, J. Z. H. Predicting Mutation-Induced Stark Shifts in the Active Site of a Protein with a Polarized Force Field. J. Phys. Chem. A 2013, 117, $6015-6023$.

(69) Zoi, I.; Antoniou, D.; Schwartz, S. D. Electric Fields and Fast Protein Dynamics in Enzymes. J. Phys. Chem. Lett. 2017, 8, 6165-6170.

(70) Shaik, S.; Ramanan, R.; Danovich, D.; Mandal, D. Structure and reactivity/selectivity control by oriented-external electric fields. Chem. Soc. Rev. 2018, 47, 5125-5145.

(71) Stuyver, T.; Danovich, D.; Joy, J.; Shaik, S. External electric field effects on chemical structure and reactivity. WIREs Comput Mol Sci 2020, 10, e1438.

(72) Ciampi, S.; Darwish, N.; Aitken, H. M.; Díez-Pérez, I.; Coote, M. L. Harnessing electrostatic catalysis in single molecule, electrochemical and chemical systems: a rapidly growing experimental tool box. Chem. Soc. Rev. 2018, 47, 5146-5164. 


\section{Graphical TOC Entry}
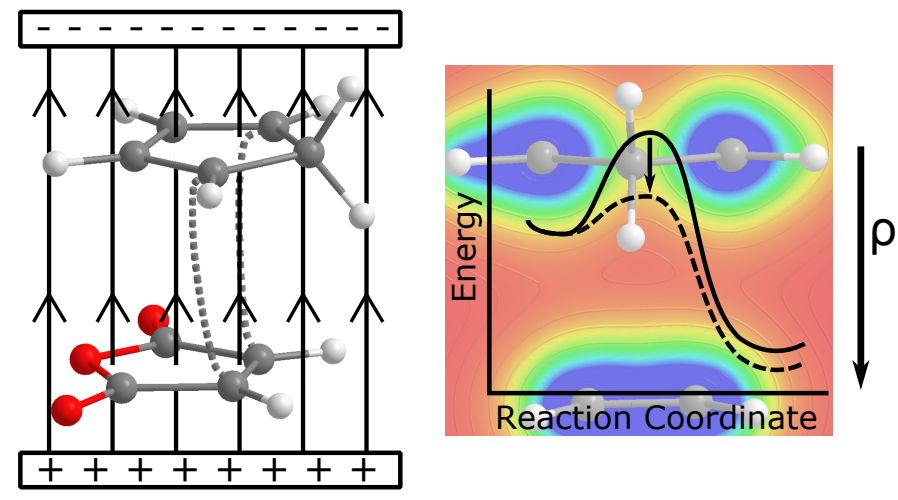